\title{
Current Status of the Response to Covid-19, Challenges and Prospects: Example of Senegal
}

\author{
Pr. Massamba DIOUF \\ ${ }^{1}$ UMI 3189 Unit Mixte International UCAD-CNRST- CNRS-USTTB-UGB Environment, Health, Society. 45391 \\ Dakar, Fann, Senegal. \\ ${ }^{2}$ Public health department /Faculty of Medicine / Cheikh Anta Diop University, Dakar (Senegal) \\ *Corresponding Author: Pr. Massamba DIOUF, Unit Mixte International UCAD-CNRST- CNRS- \\ USTTB- UGB UGB Environment, Health, Society. 45391 Dakar, Fann, Senegal, Email: \\ dioufmass78@yahoo.fr
}

\begin{abstract}
Objectives

The objective of this work is to analyze at mid-term the current state of the response to Covid-19 in Senegal and to identify challenges and prospects.
\end{abstract}

\section{Study Design}

This is a documentary review of the various reports and press releases produced by the Ministry of Health and Social Action of Senegal from 2 March 2020 (date of the index case) to 7 May 2020.

\section{Method}

The search was manual and electronic at the same time. All documents on the status of the epidemiological response, as well as communiqués and statements from the Ministry of Health, its directorates and other sectors involved in the fight were collected since the beginning of the epidemic in Senegal. The key words used were "response", "Covid-19", "Senegal" combined with Boolean operators.

\section{Results}

The current status of the response shows, despite the strategies and measures that 33 health districts out of 79 are affected in 22 departments and 11 out of 14 regions. Out of 17,450 tests carried out, 1,433 are positive ( $8.21 \%), 493$ recovered $(34.40 \%)$ and 12 deaths ( $0.84 \%$ lethal).

\section{Discussion}

The challenges and prospects reveal that there is a need to readjust the control strategy with regard to asymptomatic cases resulting from community transmission by favoring the random sampling method at the level of covidogenic neighborhoods as in demographic and health surveys, the management of the flow of asymptomatic or paucisymptomatic patients in out-of-hospital as well as in university residences, and the relaxation of measures with a socio-economic impact such as trade and the education sector.

\section{Conclusion}

The strategies need to be readjusted and then a relaxation of the prohibition measures should be considered. This relaxation would make it possible to oxidize a sector such as the economy in a context of underdevelopment.

Abbreviations: ETCs: epidemic treatment centers

Keywords: Covid-19; Response, Challenges, Senegal

\section{INTRODUCTION}

Almost 70 days ago, Senegal adopted a response strategy against Covid-19 which, although it has produced interesting results, has not always succeeded in containing the epidemic (1).

Epidemiological surveillance, instead of being dynamic, seems static with the investigation of suspected and confirmed cases, the follow-up of contact cases and the screening of asymptomatic cases that have frequented level 3 health facilities. These findings are also noted in the communication with the same style of communication using the same media with the same actors. However, the epidemic is in the active phase and is spreading with great speed, 
affecting more than thirty districts in more than twenty departments in more than ten regions against a backdrop of cases resulting from community transmission (2). The latter have so far only been detected when they are symptomatic. These Community cases are quietly concealed in the general population keeping the contamination alive. Covid-19 disease regularly brings new knowledge on a clinical, diagnostic, therapeutic and epidemiological level. Any truth of today can easily become obsolete tomorrow. Should we then continue to retaliate or should we not launch the great offensive against Covid-19? The aim of this work is to analyze the current state of the response to the Covid-19 in Senegal at the half-way point and to identify the challenges and prospects.

\section{MATERials \&MethodS}

This is a documentary review of the various reports and press releases produced by the Ministry of Health and Social Action of Senegal from 2 March 2020 (date of the index case) to 7 May 2020. The search was manual and electronic at the same time. All documents on the status of the epidemiological response, as well as communiqués and statements from the Ministry of Health, its directorates and other sectors involved in the fight were collected since the beginning of the epidemic in Senegal. The key words used were "response", "Covid-19", "Senegal" combined with Boolean operators.

A total of seventeen situation reports on the epidemiological response and sixty-nine press releases were used. The information collected was related to epidemiological surveillance, patient care and institutional communication in relation to the Covid-19 pandemic in Senegal.

\section{RESULTS}

The analysis of the documents revealed that epidemiological surveillance revolved around the screening of incoming travelers, particularly those from countries with active epidemics, the training of service providers on alert and extension, followed by the upgrading of epidemic treatment centers (ETCs), the development and updating of COVID-19 procedures, the investigation of suspected and confirmed cases, the follow-up of contacts in hotel reception areas, and the screening of suspected cases and other patients in other hospital departments (3).

Twenty ETC sites have been set up, eleven of which are in Dakar, the capital, two in Touba, the second largest city demographically and economically, and one site for the other affected regions, most of them with intensive care equipment (4). Management protocols were available with certain treatments such as hydroxychloroquine and the start of out-ofhospital management of simple and asymptomatic cases.

Communication consisted of daily press releases, dissemination of awareness-raising materials and radio and television programs, press briefings and a monthly review of response activities.

All of these strategies were supported by institutional measures such as states of emergency, closure of borders, mosques, schools, banning of inter-city travel, and widespread wearing of masks in addition to barrier gestures (5).

Despite these strategies and measures, 33 districts out of 79 are affected in 22 departments and 11 regions out of 14 . Out of 17,450 tests carried out, 1,433 are positive $(8.21 \%), 493$ are cured $(34.40 \%)$ and 12 deaths $(0.84 \%$ lethal $)$ (3).

\section{DisCUSSION}

It is therefore useful to readjust control methods and strategies. Field epidemiology stipulates that a control strategy must be dynamic, all the more so as with Covid-19, one of the particularities is its mutagenic nature with surprises and changes from all points of view.

It is urgent to make major mutations in the response at the global level, in order to avoid suffering from the disease.

A weekly review instead of a monthly one would make it possible, with objectively verifiable indicators and means of verification, to assess the gaps. This implies that weekly objectives should be set in relation to the reduction of incident cases, the appropriation of certain barrier measures and the observance of rules for both patients and health personnel in both hospital and out-of-hospital settings. These objectives must be formulated for each area taking into account specificities such as the existence of cases resulting from community transmission.

The strategy for these cases needs to be readjusted. The solution is no longer to wait for signs of this type of case to appear before detecting and questioning it to know that it is not traceable. It is appropriate to launch an 
offensive against asymptomatic community cases by favoring a random sampling approach at the level of "covidogenic" neighborhoods in order to test, detect, treat and break the chain of transmission at the same time. This approach is technically and financially feasible and is used in demographic and health surveys in our countries for the population census. These types of surveys are even recommended by some authors in the fight against Covid-19 (6).

The flow of patients that would result from these approaches would be managed by pooling private health structures and university campuses for simple to moderate cases by involving students at the end of the cycle from the various Senegalese faculties of medicine and any other person with knowledge of health, after of course an appropriate upgrading. All these strategies combined with barrier measures and rules, which, incidentally, are extremely important, would help to limit the spread of cases resulting from community transmission.

The data from these random methods would at the same time make it possible to feed compartmentalized mathematical prediction models with contextual variables that can estimate the basic reproduction rate with a low risk of error. This rate, called R0, is the expected number of new cases of an infection caused by an infected individual only in a contact population.

The mapping of the epidemic in Senegal also requires us to envisage as soon as possible a readjustment of institutional measures with regard to the negative externalities resulting from restrictions in order to avoid parallel pandemics on the medical, economic, and social and other levels. For example, regions with zero cases, as well as those that have recorded a few sporadic cases, must benefit from special strategies for relaxation while rigorously monitoring the various points of entry or exit from these localities and setting up sentinel sites at the community level with more responsibilities conferred on the populations.

This easing must also be extended to certain places of worship and other businesses with strict supervision and respect for barrier measures and gestures such as the systematic wearing of masks and physical distancing. It should be noted that the use of imams or bishops as intermediaries has always made a considerable contribution to raising awareness. In times of crisis, the appearance, from time to time, of the Head of State and other temporal and spiritual leaders can ease the atmosphere, boost the morale of the troops and strengthen the confidence of the population. Their images or speeches in these crucial moments of pandemic, can help to preserve cohesion and unity in the face of the Covid-19.

\section{CONCLUSION}

The strategies need to be readjusted and then a relaxation of the prohibition measures should be considered. This relaxation would make it possible to oxidize a sector such as the economy in a context of underdevelopment.

\section{ACKNOWLEDGEMENTS}

We thanks the chief of the department of data in the healthministry of Senegal

\section{REFERENCES}

[1] Ministry of Health and Social Action. Response to the COVID-19 coronavirus epidemic, Senegal. Situation Report $\mathrm{N}^{\circ} 17$ of 4 May 2020http://www.sante.gouv.sn/sites/default/files/ SITREP\%2017\%20Covid-19\%2004-052020.pdf

[2] Ministry of Health and Social Action. Response to the COVID-19 coronavirus epidemic, Senegal. Situation Report $\mathrm{N}^{\circ} 16$ of 30 April 2020http://www.sante.gouv.sn/sites/default/files/ SITREP\%2016\%20Covid-19\%2030-042020.pdf

[3] Ministry of Health and Social Action. Response to the COVID-19 coronavirus epidemic, Senegal. Situation Report $\mathrm{N}^{\circ} 18$ of 7 May 2020http://www.sante.gouv.sn/sites/default/files/ SITREP\%2018\%20Covid-19\%2007-052020.pdf

[4] Ministry of Health and Social Action. Response to the COVID-19 coronavirus epidemic, Senegal. Situation Report $\mathrm{N}^{\circ} 3$ of 12 March 2020http://www.sante.gouv.sn/sites/default/files/ SITREP\%203\%20Covid-19\%2012-03-2020.pdf

[5] Ministry of Health and Social Action. Response to the COVID-19 coronavirus epidemic, Senegal. Situation Report $N^{\circ} 10$ of 6 April 2020http://www.sante.gouv.sn/sites/default/files/ SITREP\%2010\%20Covid-19\%2006-042020.pdf

[6] Subramanian SV, James KS. Use of the Demographic and Health Survey framework as a population surveillance strategy for COVID-19. Lancet Glob Health. 8,1(2020) https://doi.org/ 10.1016/ S2214-109X(20)30213-8 
Citation: Massamba DIOUF. Current Status of the Response to Covid-19, Challenges and Prospects: Example of Senegal .ARC Journal of Public Health and Community Medicine. 2020; 5(4):30-33. Doi:doi.org/10. 20431/24 56-0596.0504005.

Copyright: (C) 2020 Authors. This is an open-access article distributed under the terms of the Creative Commons Attribution License, which permits unrestricted use, distribution, and reproduction in any medium, provided the original author and source are credited. 